\title{
Psychophysical exponents for single papillae: A comparison with whole-mauth exponents
}

\author{
ARMAND V. CARDELLO \\ Behavioral Sciences Division, Food Sciences Laboratory \\ U.S. Army Natick Research \& Development Command, Natick, Massachusetts 01760
}

\begin{abstract}
Taste intensity judgments in response to chemical stimulation of single human fungiform papillae were obtained by the method of magnitude estimation. Intensity scales constructed from these data were compared to those obtained from the same subjects in response to stimulation of the whole mouth. Single papilla functions conformed well to the power law governing the growth of sensation magnitude, although a minority of single papilla functions exhibited peaking of response magnitude at intermediate concentrations, followed by a decline in response magnitude at higher concentrations. Single papilla exponents were found to be lower than whole mouth exponents and were positively correlated with detection and/or recognition thresholds of the papillae for most compounds. Exponents of summated single papilla functions were greater than median single papilla exponents, but their relative magnitude vs. whole mouth exponents varied by test compound. The data were discussed in relationship to previous findings concerning flow rate effects, threshold-exponent relationships across the tongue surface, and level-dependent spatial summation.
\end{abstract}

Intensity functions obtained by ratio scaling procedures support the view that perceptual magnitude grows as a power function of the physical intensity of the stimulus. The exponents of these functions have been interpreted by Stevens (1970, 1971) to be constants that reflect basic transducer properties of the receptors. This "sensory transducer theory" suggests that the power transformation of stimulus energy occurs at the extreme periphery of the nervous system and that, once transformed into afferent neural energy, all subsequent transformations of energy are linear.

Although the notion of exponent invariance is theoretically appealing, considerably variation has been found in the magnitude of the exponents for a number of sensory attributes. An inverse relationship has been demonstrated between the intramodal stimulus range and the value of the power exponent by Engen (1956), Engen and Levy (1958), Jones and Woskow (1966), and Poulton (1968) in numerous psychophysical experiments. It has also been demonstrated in a number of sensory systems that the area of stimulation can affect the power exponent through level-dependent spatial summation (see Marks, 1974). However, in the gustatory system, the evidence for this is contradictory. Bujas and Ostojcic (1941), Hara (1955), and Smith (1971) have each

This research was conducted as part of a thesis submitted in partial fulfillment of the degree of Doctor of Philosophy at the University of Massachusetts/Amherst. The author wishes to express his deepest appreciation to Dr. Ernest Dzendolet for his guidance and support during the conduct of this research and to Drs. Owen Maller, Claire Murphy, and Harry Lawless for their comments on the manuscript. reported spatial summation of response magnitude for taste. Bujas and Ostojcic's (1941) data show that the degree of summation is dependent upon sensation level, with less summation occurring at higher sensation levels, but Smith's (1971) data show no significant change in exponents for $\mathrm{NaCl}$, citric acid, quinine hydrochloride, or saccharin for tongue areas ranging from 4 to $126 \mathrm{~mm}^{2}$.

Less ambiguous than the data on level-dependent spatial summation are the data demonstrating the effects of locus stimulation and stimulus presentation procedure on taste exponents. Collings (1974) has shown that the magnitude of taste exponents varies across the tongue surface and that the relative magnitude by location is dependent upon the particular test compound. Bartoshuk and Cleveland (1977) and Meiselman (1971) have also shown systematic variation in taste exponents as a function of stimulus presentation procedures. These latter data show standard sip procedures to produce higher exponents than dorsal flow procedures (Meiselman, 1971). Meiselman and Bose (1977) and Meiselman, Bose, and Nykvist (1972) have further shown that taste exponents for sip and flow procedures may be related to differences in flow rate between the two procedures.

From the perspective of the above effects on exponent values, the technique of chemically stimulating single human taste papillae is of theoretical interest. The reason for this is that the technique involves the stimulation of small and highly localized areas on the surface of the tongue and involves the use of a unique stimulus presentation procedure. Unfortunately, previous reports of the sensory 
responses elicited from single taste papillae have only focused on the question of taste quality specificity (Bealer \& Smith, 1975; Békésy, 1966; Cardello, 1978; Harper, Jay, \& Erickson, 1966; Kuznicki, 1978; McCutcheon \& Saunders, 1972). Relatively little information is available concerning the growth of perceived intensity in these papillae. Both McCutcheon and Saunders (1972), using a 5-point scale, and Kuznicki (1978), using magnitude estimation, obtained intensity judgments in response to stimulation of single fungiform papillae. However, both used only a single concentration of each test compound, thus precluding construction or analysis of concentration-response curves.

The present report focuses on the shape of the suprathreshold concentration-response curves of single papillae and compares them with those obtained by stimulation of the whole mouth. In addition, the relationship between these functions and individual taste thresholds of the papillae are assessed, in order to provide information about the sources of variability in exponents and to aid in understanding the mechanism by which intensity information is integrated over the surface of the tongue.

\section{METHOD}

Subjects

The subjects were two males and two females between the ages of 18 and 25 years. Each subject was screened for participation by the method reported in Meiselman and Dzendolet (1967), and each was requested not to eat, drink, or smoke for $1 \mathrm{~h}$ preceding each test session.

\section{Apparatus and Stimuli}

The apparatus for stimulation of single papillae consisted of a series of disposable plastic 1-ml tuberculin syringes, fitted with 33-ga., blunt, stainless steel hypodermic needles. Droplets of test solution were presented to the dorsal surface of fungiform papillae with the aid of a dissecting microscope (see Cardello, 1978, for a detailed description of the apparatus and the stimulating procedure).

The test solutions used for both single papilla and whole mouth stimulation appear in Table 1. All stimuli were prepared with reagent grade chemicals and were mixed with distilled water. All solutions were tested at room temperature $\left(25^{\circ} \mathrm{C}\right)$.

\section{Procedure}

Single papillae. The subject sat adjacent to a sink. At the start of each trial, the subject extended his/her tongue to expose the anterior $3 \mathrm{~cm}$. For the next $45 \mathrm{sec}$, the tongue remained extended in room atmosphere while excess saliva was allowed to evaporate from its surface. A .05- $\mu$ l droplet of solution was then presented to the dorsal surface of a test papilla. Ten papillae were tested quasirandomly in each subject, with successive presentations to the same papilla separated by at least five presentations to other papillae.

The subjects made two judgments of the stimulus while the tongue remained in an extended position. The first judgment was of its taste quality, and the second was of its perceived intensity. Quality choices included salty, sweet, sour, bitter, indistinct or vague, no taste, and complicated taste. Intensity judgments were made by the method of magnitude estimation. No modulus was used, and "no taste" responses were assigned a magnitude estimate of zero.

Since no modulus was used and testing continued over a period of 4 months, provisions were made for normalizing the magnitude estimates across sessions. To accomplish this, four "standard" solutions were presented during each session. The standard solutions were those asterisked in Table 1. Each of these solutions was presented twice to the same papilla during each session.

After making each set of judgments, the subject rinsed his/her tongue with distilled water, retracted it, and awaited the next trial. A 2-min interstimulus interval was used, and each solution was presented twice to each papilla. Although solutions were presented by the method of constant stimuli, the method was modified slightly to reduce the number of subthreshold solutions that were presented (for details, see Cardello, 1978)

Whole mouth. Whole mouth testing was conducted at the completion of all single papilla tests. At the start of each trial, the subject was presented with $2 \mathrm{ml}$ of test solution in a plastic cup. He was instructed to sip the entire contents of the cup, hold the solution in his/her mouth for $3 \mathrm{sec}$ and then expectorate. The subject reported the taste quality of the solution and gave a magnitude estimate of its intensity. After making his/her response, the subject rinsed with distilled water and awaited the next trial. A 3-min ISI was employed. Solutions were presented randomly, and each was presented only once. The latter procedure was adopted in order to reduce the number of solution presentations and to minimize adaptation effects.

Table 1

Test Solu tions

\begin{tabular}{|c|c|c|c|c|c|c|c|c|}
\hline Sucrose & Dextrose & $\begin{array}{l}\text { Quinine } \\
\text { Sulfate }\end{array}$ & $\begin{array}{l}\text { Quinine } \\
\text { Hydrochloride } \\
\text { (Monohydrate) }\end{array}$ & $\mathrm{HCl}(\mathrm{pH})$ & $\begin{array}{c}\text { Citric } \\
\text { Acid }(\mathrm{pH})\end{array}$ & $\mathrm{NaCl}$ & $\mathrm{LiCl}$ & $\mathrm{KCl}$ \\
\hline 1,000 & 1,500 & 3 & 100 & $50(1.30)$ & $500(1.66)$ & 5,000 & 5,000 & 3,500 \\
\hline $700 *$ & 1,000 & $2 *$ & 50 & $40(1.40)$ & $250(1.83)$ & 3,500 & 3,500 & 2,000 \\
\hline 500 & 500 & 1 & 25 & $30(1.52)^{*}$ & $100(2.06)$ & $2,000^{*}$ & 2,000 & 1,000 \\
\hline 250 & 250 & .5 & 10 & $20(1.70)$ & $50(2.24)$ & 1,000 & 1,000 & 500 \\
\hline 100 & 100 & .25 & 5 & $10(2.00)$ & $25(2.45)$ & 500 & 500 & 250 \\
\hline 50 & 50 & .10 & 2.5 & $5(2.30)$ & $10(2.62)$ & 250 & 250 & 100 \\
\hline 25 & 25 & .05 & 1.0 & $1(3.00)$ & $5(2.81)$ & 100 & 100 & 40 \\
\hline 10 & 10 & .025 & .5 & $.5(3.30)$ & $2.5(2.98)$ & 40 & 40 & 10 \\
\hline \multirow[t]{4}{*}{5} & 5 & .01 & .25 & $.25(3.60)$ & $1.0(3.20)$ & 10 & 10 & 5 \\
\hline & 2.5 & & .1 & $.1(4.00)$ & $.5(3.36)$ & 5 & 5 & 2.5 \\
\hline & & & .05 & & $.25(3.53)$ & 2.5 & 2.5 & \\
\hline & & & & & & 1.0 & 1.0 & \\
\hline
\end{tabular}

Note-All solution concentrations are expressed in millimoles. 


\section{RESULTS}

\section{Single Papilla Data}

Magnitude estimates were normalized across subjects and sessions. Geometric means of the magnitude estimates were calculated, except in those cases where zeros were present. In such cases (approximately $10 \%-15 \%$ ), medians were calculated. The geometric mean (or medians) of the magnitude estimates were plotted as a function of concentration for each papilla, solution, and subject. A total of $\approx 260$ psychophysical functions were constructed. Three representative functions for each of the nine test compounds are shown in Figure 1. All are plotted in full logarithmic coordinates.

All single-papilla response curves that comprised at least five data points were fitted by linear, logarithmic, and power functions, using a leastsquares regression procedure. Correlation coefficients were converted to $\mathrm{Z}$ scores, and a two-way repeated-measures analysis of variance was performed to determine if either the compound or the function fitted to the data had an effect on the regression coefficients. Results of the analysis indicated no effects due to either variable, although the relative magnitude of median $r$ values suggested that a power function $(r=.71)$ provided a somewhat better description of the data than either a linear $(r=.63)$ or a logarithmic $(r=.65)$ function.

The exponents of the best-fitting power functions were calculated from the above data and the median exponents for each compound appear in Column A of Table 2.

In order to maintain statistical precision in the estimation of exponent values, the exponents in Column A of Table 2 were based only on data from those papillae whose psychophysical functions comprised five or more data points. Thus, operationally, only those papillae that mediated responses to the five most concentrated solutions of a compound were included in the analysis. Papillae that mediated responses to fewer concentrations were not included, thereby biasing the sample with a disproportionate number of papillae having low thresholds. In order to assess the representativeness of the exponents shown in Column A, power functions were fitted to all concentration-response curves comprised of at least two data points. The median exponents for these data appear in Column B of Table 2. For each compound, the median exponent of this expanded population of papillae is greater than for the subset of papillae represented in Column A.

In order to test for the existence of a correlation between exponents and thresholds (a possibility suggested by the above difference in exponents between Columns A and B), product-moment correlation coefficients were calculated between the obtained exponents and the detection and recognition thresholds of individual papillae. Detection thresholds were calculated for each papilla by determining the concentration of each compound that was detected $50 \%$ of the time. The threshold concentrations were extrapolated from a least-squares linear regression line determined between the last concentration at which there were $100 \%$ "no taste" responses and the first concentration at which there were $100 \%$ "indistinct or vague" and/or other tastequality responses. Recognition thresholds were determined in an analogous manner but were defined as the concentration of each compound that was recognized as having a true taste quality $50 \%$ of the time (see Cardello, 1978).

The obtained correlation coefficients between exponents and thresholds appear in Table 3. All coefficients are positive, and 15 of the 18 are statistically significant. In the case of the citric acid exponents, neither the correlation with detection thresholds nor that with recognition thresholds is significant. For dextrose, the correlation with recognition thresholds is also not significant.

\section{Summated Single Papilla Data}

Summated response functions (the integrated response across all papillae) were determined for each compound and subject by summing the magnitude estimates for all 10 papillae at each concentration. The median exponents of these summated functions appear in Column $\mathrm{C}$ of Table 2. It can be seen that the exponents of these functions are all greater than the median single-papilla exponents (Column B).

\section{Whole-Mouth Data}

Magnitude estimates for whole-mouth responses were normalized and the data for each subject and compound were fitted by linear, logarithmic, and power functions. Like the results for single papillae, analysis of variance showed no significant differences among the functions fitted to the data, although the median $r$ values again suggested that a power function provided the best description of the data.

Median exponents across subjects, determined for the best-fitting power functions, appear in Column D of Table 2. A two-way repeated-measures analysis of variance showed a significant difference in exponents between the single-papilla and wholemouth functions $(\mathrm{F}=12.54, \mathrm{df}=1 / 3, \mathrm{p}<.05)$ and among compounds $(F=4.16, \mathrm{df}=8 / 24, \mathrm{p}<.05)$.

\section{DISCUSSION}

Some of the single papilla functions shown in Figure 1 peak at high or intermediate concentrations 

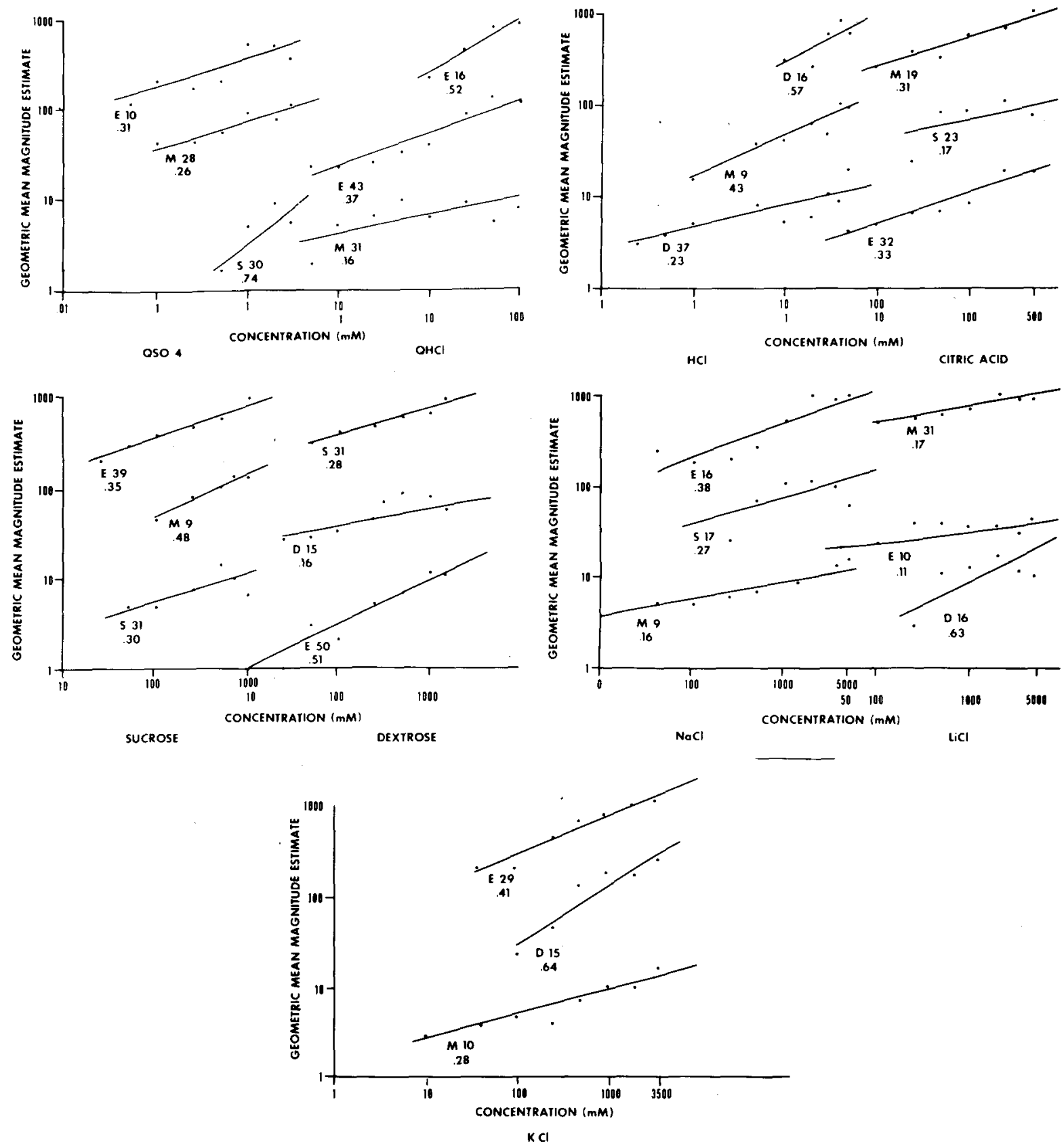

Figure 1. Representative psychophysical functions for single human fungiform taste papillae. The letter-number code below each curve designates the subject and the papilla from which the data were obtained. The numerical value below each code is the exponent for the best-fitting power function.

and then decline in magnitude. Examples of papillae characterized by such intensity curves are S-31 to sucrose, D-15 to dextrose, and $\mathrm{S}-17$ to $\mathrm{NaCl}$. The shapes of these functions are similar to electrophysiological concentration-response functions obtained from single fungiform papillae in the rat (Miller, 1971). It has been suggested that such response peaking, termed "overload" by Pfaffman (1955), results from antidromic inhibition (Miller,
1971). Similar nonlinearities were not observed in any of the whole-mouth functions, reflecting the fact that the number of papillae exhibiting such response functions are few in number $(\approx 10 \%)$ and have only a small net effect on the whole-mouth response.

In addition to peaking, a minority of single papilla functions approached asymptote at high concentrations, but did not decline with further increases in concentration. It is uncertain whether these functions 
Table 2

Median Exponents of the Best-Fitting Power Functions for (A) Single Papilla Functions Comprised of Five or More Data Points, (B) Single Papilla Functions Comprised of Two or More Data Points, (C) The Summated Single Papilla Functions, and (D) Whole-Mouth Functions

\begin{tabular}{lcccc}
\hline & $\begin{array}{c}\text { (A) } \\
\text { Single Papillae } \\
\text { Solution }\end{array}$ & $\begin{array}{c}\text { (B) } \\
\text { Single Papillae }\end{array}$ & $\begin{array}{c}\text { (C) } \\
\text { Summated Single } \\
\text { Papilla Function }\end{array}$ & $\begin{array}{c}\text { (D) } \\
\text { Whole-Mouth } \\
\text { Function }\end{array}$ \\
\hline Sucrose & .22 & .36 & .55 & .61 \\
Dextrose & .28 & .37 & .51 & .93 \\
Quinine Sulfate & .17 & .40 & .48 & .47 \\
Quinine Monohydrochloride & .28 & .30 & .45 & .42 \\
HCl & .41 & .67 & 1.24 & .44 \\
Citric Acid & .34 & .38 & .59 & .33 \\
NaCl & .18 & .21 & .42 & .68 \\
LiCl & .19 & .31 & .41 & .66 \\
KCl & .38 & .45 & .66 & .74 \\
\hline
\end{tabular}

would have also exhibited a response decrement if tested with concentrations higher than were used in this study.

Although not significantly larger, the median $r$ values for the fit of power functions to the single papilla data indicate that the psychophysical response functions for individual papillae conform well to the power law governing the growth of sensation magnitude. The failure to find significant differences among the fitted functions is most likely related to the response peaking observed in some of the curves. If data points at the highest concentrations are ignored, the remaining points fall more nearly along a straight line in full logarithmic coordinates. While this would improve the fit of a power function to these data, it would require the elimination of data that are important to the analysis of the summated response functions. Elimination of these data would unduly confound comparisons of single-papilla and summated response exponents, since they would no longer be based on comparable sets of data.

The data in Table 3 reveal positive correlations between stimulus thresholds and suprathreshold

Table 3

Pearson Product-Moment Correlation Coefficients (r) Between the Obtained Psychophysical Exponents and the Absolute and Recognition Thresholds of Individual Fungiform Papillae

\begin{tabular}{lll} 
& $\begin{array}{c}\text { Correlation With } \\
\text { Absolute Threshold }\end{array}$ & $\begin{array}{c}\text { Correlation With } \\
\text { Recognition Threshold }\end{array}$ \\
\hline Sucrose & $.67^{*}$ & $.60^{*}$ \\
Dextrose & $.46^{* *}$ & .34 \\
Quinine Sulfate & $.67^{*}$ & $.72^{*}$ \\
Quinine & & \\
Monohydrochloride & $.59^{*}$ & $.74^{*}$ \\
HCl & $.64^{*}$ & $.44^{*}$ \\
Citric Acid & .19 & .23 \\
$\mathrm{NaCl}$ & $.76^{*}$ & $.75^{*}$ \\
$\mathrm{LiCl}$ & $.90^{*}$ & $.88^{*}$ \\
$\mathrm{KCl}$ & $.45^{*}$ & $.46^{*}$ \\
\hline
\end{tabular}

$* p<.01 ; * *<.05$. psychophysical exponents within single papillae. In the case of sucrose and quinine monohydrochloride, these correlations are consistent with the data of Collings (1974), who used saturated filter paper to determine both recognition thresholds and intensity functions for four loci on the tongue and on the soft palate. Collings (1974) reported a significant positive correlation between threshold (negative correlation for sensitivity) and size of exponent for these compounds. However, in contrast to Collings (1974), who found no correlation between thresholds and exponents for $\mathrm{NaCl}$, the observed correlation for $\mathrm{NaCl}$ in this study was $\approx .75$. Similarly, Collings (1974) reported a negative correlation between thresholds and exponents for citric acid, while a nonsignificant positive correlation was found for citric acid in the present study.

The positive correlations between thresholds and exponents found in the present study underlie the observed differences between the median single papilla exponents of Columns A and B in Table 2. The sampling bias that allowed only those papillae with low thresholds to be included in the data base of exponents in Column $A$ also resulted in a sample of papillae with correspondingly low exponents. In view of the fact that the exponents in Column $B$ are based on a more representative sample of papillae, all subsequent discussions of exponents for single papillae will be with reference to the values shown in Column B.

For all compounds, the median exponents of the summated response functions (Column C) are larger than the median single papilla exponents (Column B). One likely explanation of this difference involves the observed correlations between thresholds and exponents. Simply stated, at lower stimulus concentrations only those papillae with low thresholds contribute to the summated response. Since these papillae are characterized by smaller exponents, the growth of perceived intensity is slow at these low concentrations. At higher concentrations, papillae 
with higher thresholds, and correspondingly higher exponents, contribute to the summated response. The combined contribution of an increased number of responding papillae at higher concentrations and the greater slopes (exponents) of these high-threshold papillae have the effect of producing a higher slope for the summated function.

It should be kept in mind that the summated response functions discussed above were constructed from the responses of papillae that were spatially nonadjacent and that were stimulated sequentially. Thus, the effects of peripheral neural interactions are not reflected in these functions. However, the fact that the exponents for the summated functions are larger than the median exponents for single papillae indicates the importance to the whole-mouth response of integrated responding from a population of papillae with separate and distinct thresholds.

Although the whole-mouth exponents in Column D of Table 2 are smaller than those usually cited in the literature, only the two sugar functions approached an asymptote at high concentrations. Thus, the lower slopes of these functions must be attributed to either the large range of stimulus concentrations used with each compound or the particular subject sample. In either case, since the single papilla and whole-mouth data were obtained with the same stimuli and the same subject sample, a common basis exists for comparison of the data.

One hypothesis that might be put forward to explain the fact that the median single papilla exponents (Column B) are lower than those obtained from the whole mouth (Column D) is based on differences in flow rate between the two stimulating procedures, i.e., the flow rate of a stimulus droplet placed on the dorsal surface of a papilla is likely to be extremely small compared to that for a sipped stimulus. However, since increased flow rate is assumed to affect taste exponents by an increase in rate of stimulation at the receptor, the direction of change in exponent with flow rate would be expected to be the same for all compounds. This is not the case in these data, since, in contrast to the other compounds, the acids have higher single-papilla exponents (Column B) than whole-mouth exponents.

An alternative hypothesis is that the observed differences in exponents for single-papilla and wholemouth stimulation are attributable to spatial summation across different classes of papillae, each of which possesses a different threshold for each of the various taste compounds, and each of which also possesses exponent values that are positively correlated with these thresholds.

Such an explanation would be compatible with the data of Collings (1974), who reported that the lowest tongue thresholds for sucrose, $\mathrm{NaCl}$, urea, and quinine hydrochloride were at the front of the tongue (fungiform papillae), while for citric acid, the lowest tongue thresholds were on the sides (foliate papillae). Since only fungiform papillae were examined in the present study, the observed higher single-papilla exponents for citric acid and $\mathrm{HCl}$ may be due to the fact that fungiform papillae have relatively higher thresholds and higher exponents for acids than do papillae at other tongue loci.

Similarly, a comparison of Columns C and D in Table 2 reveals that the exponents of the summated functions for sugars and salts, while greater than median exponents for single papillae, are lower than those for the whole mouth. In contrast, the bitter exponents are near whole-mouth magnitudes and the acid exponents are greater than their whole-mouth counterparts. These differences may be explained by the fact that the summated functions are composites of responding from only fungiform papillae, while the whole-mouth curves reflect the responses of additional tongue loci. In the case of salts and sugars, which have lower thresholds at fungiform loci (Collings, 1974), positively correlated exponents would account for the lower exponents of the summated single-papilla curves. For the bitter compounds, a similar explanation would hold, although the difference. between the thresholds at fungiform and other loci is much smaller. For the acids, which have higher thresholds at fungiform loci, the explanation afforded by correlated exponents is again sufficient to account for the higher exponents.

The fact that Smith (1971) did not observe changes in exponents with increases in area of stimulation may have been due to the fact that his smallest area of stimulation $\left(4 \mathrm{~mm}^{2}\right)$ involved a large enough number of papillae so that the total response of these papillae produced exponents representative of fungiform loci (note that the summated response of just 10 papillae in this study significantly increased the exponent over single papilla values). Since Smith (1971) only varied areal extent over fungiform loci, the contribution of other papilla types (circumvallate and foliate) would not have altered these exponent values.

A comparative study of thresholds and suprathreshold functions in individual fungiform, foliate, and circumvallate papillae would provide the important information needed to assess the role of each papilla type to the whole-mouth response and to help to further clarify the mechanism(s) underlying the observed differences between single papilla and whole-mouth exponents.

\section{REFERENCES}

Bartoshuk, L., \& Cleveland, C. Mixtures of substances with similar tastes: A test of a psychophysical model of taste mixture interactions. Sensory Processes, 1977, 1, 177-186.

BeAler, S. L., \& Smith, D. V. Multiple sensitivity to chemical 
stimuli in single human taste papillae. Physiology \& Behavior, 1975, 14, 795-799.

BÉkÉsy, G. von. Taste theories and the chemical stimulation of single papillae. Journal of Applied Physiology, 1966, 21, $1-9$.

Bujas, Z., \& Ostojcic, A. La sensibilité gustative en fonction de la surface excitée. Acta Instituti Psychologia Universitatis Zagrebiensis, 1941, 13, 1-19.

Cardello, A. V. Chemical stimulation of single human fungiform taste papillae: Sensitivity profiles and locus of stimulation. Sensory Processes, 1978, 2, 173-190.

Collings, V. B. Human taste response as a function of locus of stimulation on the tongue and soft palate. Perception \& Psychophysics, 1974, 16, 169-174.

Engen, T. An evaluation of a method for developing ratioscales. American Journal of Psychology, 1956, 69, 92-95.

Engen, T., \& Levy, N. The influence of context on constantsum loudness judgments. American Journal of Psychology, $1958,71,731-736$.

HARA, S. Interrelationships among stimulus intensity, stimulated area and reaction in the human gustatory sensation. Bulletin of the Tokyo Medical \& Dental University, 1955, 2, 147-158.

HARPER, H., JAY, J., \& Erickson, R. Chemically evoked sensations from single human taste papillae. Physiology \& Behavior, 1966, 1, 319-325.

Jones, F. N., \& Woskow, M. J. Some effects of context on the slope in magnitude estimation. Journal of Experimental Psychology, 1966, 71, 170-176.

Kuznicki, J. T. Taste profiles from single human taste papillae. Perceptual \& Motor Skills, 1978, 47, 279-286.

Marks, L. Sensory processes: The new psychophysics. New York: Academic Press, 1974.
McCutcheon, N. B., \& Saunders, J. Human taste papilla stimulation: Stability of quality judgments over time. Science, 1972, 175, 214-216.

Meiseliman, H. L. Effect of presentation procedure on taste intensity functions. Perception \& Psychophysics, 1971, 10, 15-18.

Meiselman, H. L., \& Bose, H. E. Effects of flow rate on taste intensity ratings. Chemical Senses \& Flavor, 1977, 2, 515-522.

Meiselman, H. L., Bose, H. E., \& Nykvist, W. E. Effect of flow rate on taste intensity responses in humans. Physiology \& Behavior, 1972, 9, 35-38.

Meiselman, H. L., \& Dzendolet, E. Variability in gustatory quality identification. Perception \& Psychophysics, 1967, 2, 496-498.

Miller, I. J. Peripheral interactions among single papilla inputs to gustatory nerve fibers. Journal of General Physiology, 1971, 57, 1-25.

Pfaffmann, C. Gustatory afferent impulses in cat, rat, and rabbit. Journal of Neurophysiology, 1955, 18, 429-440.

Poulton, E. C. The new psychophysics: Six models of magnitude - estimation. Psychological Bulletin, 1968, 69, 1-19.

Sмгтн, D. V. Taste intensity as a function of area and concentration: Differentiation between compounds. Journal of Experimental Psychology, 1971, 87, 163-171.

Stevens, S. S. Neural events and the psychophysical law. Science, 1970, 170, 1043-1050.

Stevens, S. S. Issues in psychophysical measurement. Psychological Reviews, 1971, 78, 426-450.

(Received for publication May 15, 1978; revision accepted February 5, 1979.) 